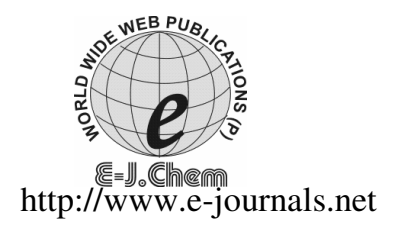

ISSN: 0973-4945; CODEN ECJHAO

E-Journal of Chemistry

2011, 8(1), 71-76

\title{
Effect of Aquo-glycolic Media and Added Anions on the Anodization of Zircaloy-4 in Sulphamic Acid
}

\author{
VIPLAV DUTH SHUKLA* and CH. ANJANEYULU \\ Department of Chemistry \\ University College for Women, Osmania University, Koti, Hyderabad, India \\ *Department of Chemistry \\ BJR Govt. Degree College, Bazarghat, Nampally, Hyderabad, India \\ drviplove@gmail.com
}

Received 31 March 2010; Revised 24 May 2010; Accepted 17 July 2010

\begin{abstract}
Anodization of zircaloy-4 in $0.1 \mathrm{M}$ sulphamic acid has been carried out. Kinetics of anodic oxidation of zircaloy-4 has been studied at a constant current density of $8 \mathrm{~mA} / \mathrm{cm}^{2}$ and at room temperature. Thickness estimates were made from capacitance data. The plots of formation voltage $v s$. time, reciprocal capacitance $v s$. time, reciprocal capacitance $v s$. formation voltage and thickness $v s$. formation voltage were drawn and rate of formation, current efficiency and differential field were calculated. The addition of solvent (ethylene glycol) showed better kinetic results. For $25 \%, 50 \%$ and $75 \%$ aquo-glycolic media, the dielectric constant values are low leading to a marked improvement in the kinetics. In $80 \%$ ethylene glycol, though the dielectric constant value of solution is less, the kinetics was slow which may be attributed to the fact that the electrolyte becomes highly non-polar. Improvement in the kinetics of oxide film formation was observed by the addition of millimolar concentration of anions $\left(\mathrm{CO}_{3}{ }^{2-}, \mathrm{SO}_{4}{ }^{2-}, \mathrm{PO}_{4}{ }^{3-}\right)$. The presence of phosphate ions improved the kinetics of anodization to better extent.
\end{abstract}

Keywords: Zircaloy-4, Anodization, Formation rate, Current efficiency, Aquo-glycolic media, Anion impurities

\section{Introduction}

Zirconium based alloys are used as structural material in water-cooled thermal reactors ${ }^{1,2}$. Zircaloy-4 is a alloy of $98 \%$ pure zirconium with other trace impurities. Zircaloy-4, due to their low cross section for thermal neutrons and because of their relatively good corrosion resistance against water and steam used in water cooled reactors. Anodization of zirconium alloys have been studied in some electrolytes ${ }^{3-6}$. 
In the present work, we report the results of our studies on the anodization of zircaloy-4 in $0.1 \mathrm{M}$ sulphamic acid and the effect of solvent (ethylene glycol) and also the effect of added anion impurities (phosphates, sulphates and carbonates). We have calculated the formation rate, current efficiency and differential field of formation of zircaloy- 4 .

\section{Experimental}

Zircaloy-4 was of $98 \%$ nominal purity, supplied in the form of annealed sheet by Nuclear Fuel Complex, Hyderabad as gift samples. The chemical composition of zircaloy-4: 0.07 wt.\% chromium; 0.23 wt.\% iron; 1.44 wt. \% tin and balance is zirconium.

In the present work, the foil samples used were cut with the aid of a punch into flag-shaped specimens of $1 \mathrm{~cm}^{2}$ working area on both side and $2 \mathrm{~cm}$ long tag. The chemical polishing mixture consisted of acids such as $\mathrm{HNO}_{3}, \mathrm{HF}$ and water in a definite volume ratio of 3:3:1.

For anodizing, a closed cell of $200 \mathrm{~mL}$ capacity was used. The cathode used was a platinum foil of $20 \mathrm{~cm}^{2}$ superficial area to make the double layer capacitance as large as possible. Electrolytes used were $0.1 \mathrm{M}$ sulphamic acid in $25 \%, 50 \%$ and $75 \%$ aquo-glycolic mixtures, the solvent being ethylene glycol and the added anionic impurities were $\mathrm{PO}_{4}{ }^{-3}$, $\mathrm{SO}_{4}{ }^{-2}$ and $\mathrm{CO}_{3}{ }^{-2}$ in $0.001 \mathrm{M}$ concentration.

All experiments were carried out at a constant current density of $8 \mathrm{~mA} \cdot \mathrm{cm}^{-2}$. The experimental procedure for the anodization is given elsewhere ${ }^{7}$. The kinetic results calculated are formation rate in $\mathrm{Vs}^{-1}$, current efficiency $(\eta) \%$ and differential fields of formation $\left(\mathrm{F}_{\mathrm{D}}\right)$ in $\mathrm{MV} \mathrm{cm} \mathrm{cm}^{-1}$ from the conventional plots $\mathrm{V}$ vs. $\mathrm{t}, 1 / \mathrm{C} v s . \mathrm{V}$ and $1 / \mathrm{c} v s . \mathrm{t}$.

\section{Results and Discussion}

Anodization of zircaloy-4 was done in various electrolytes (acidic, basic and neutral). The formation rate, the current efficiency and the differential fields were calculated.

\section{Effect of substitution}

The kinetics of zircaloy-4 anodization in $0.1 \mathrm{M}$ sulphamic acid was better. The effect of solvent and added anionic impurities was studied in $0.1 \mathrm{M}$ sulphamic acid to check whether there was enhancement in the kinetics of film formation ${ }^{8,9}$.

\section{Effect of solvent}

Anodization of zircaloy-4 in $0.1 \mathrm{M}$ sulphamic acid was performed by mixing various proportions of ethylene glycol to the aqueous solution $(25 \%, 50 \%$ and $75 \%)$. There was an improvement in the kinetics as given in Table 1. The relevant plots are shown in Figures $1 \& 2$. Aquo-organic solutions aid in the formation of good oxide films and act as better electrolytic capacitors ${ }^{10}$. These facts support the current results obtained in aquo-organic mixtures of $0.1 \mathrm{M}$ sulphamic acid. It can be explained on the basis of decrease in the dielectric constant of the medium (Table 2).

In solutions of low dielectric constant there is less chance of ion-dipole interactions (solvent-ion interactions), which do not interfere in the oxide film formation. However, the ions in the high dielectric constant solutions, interact with oxide ions responsible for oxide film formation due to high salvation with water molecules. In such solutions, the kinetics are poor. The kinetics are better in low dielectric constant solutions. For 25\%, 50\% and 75\% aquoglycolic media, the dielectric constant values are low leading to a marked improvement in the kinetics ${ }^{11}$. The oxide film formed on the zircaloy- 4 consists of two discrete layers in $0.1 \mathrm{M}$ sulphamic acid were disappeared and made to a single layer in presence of ethylene glycol. 


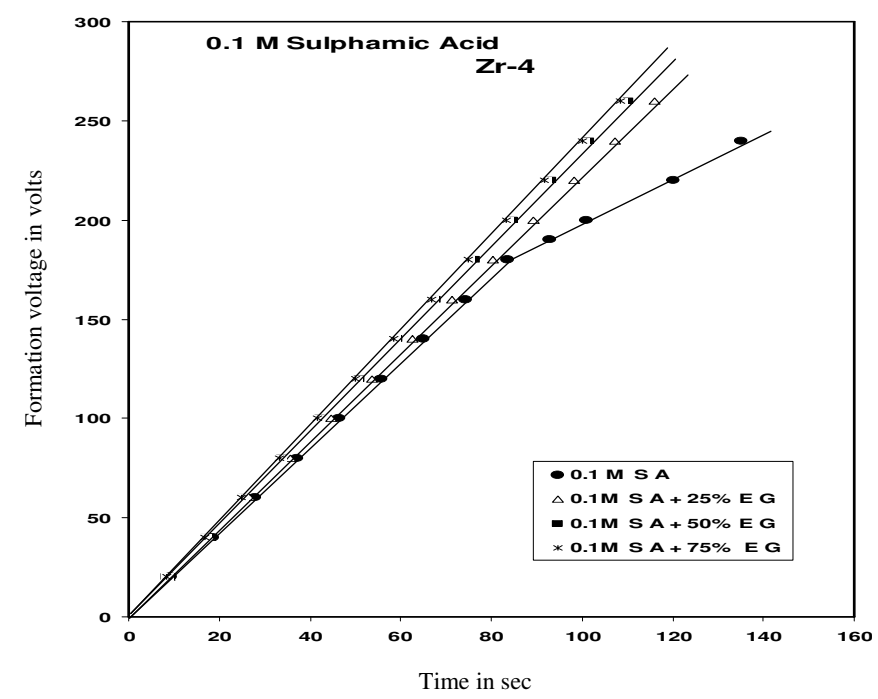

Figure 1. Plot of formation voltage as a function of time in aquo-glycolic solution

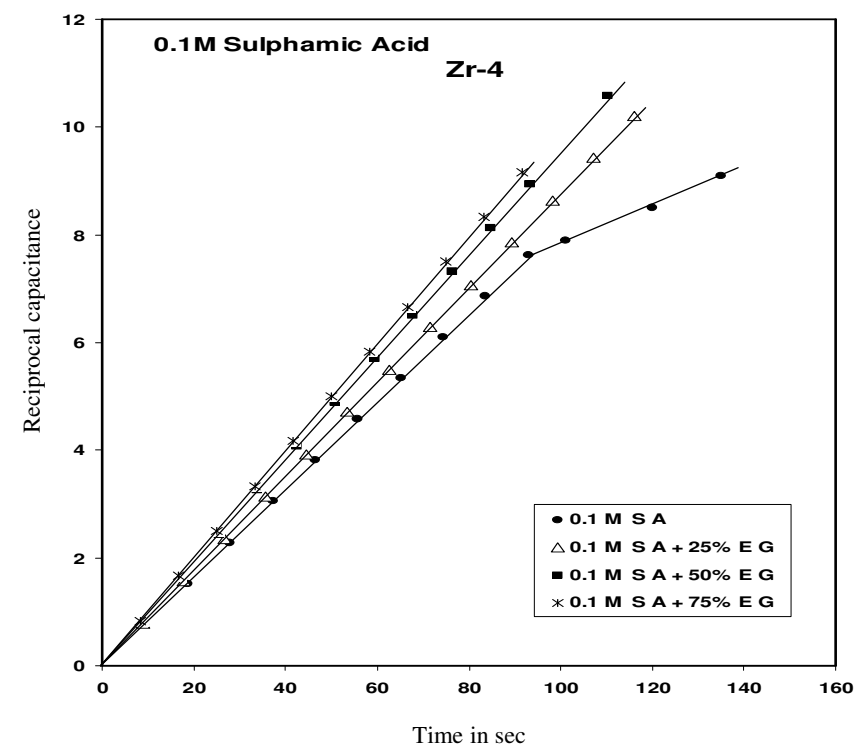

Figure 2. Plot of reciprocal capacitance as a function of time in aquo-glycolic solution

Table 1. Effect of solvent on the anodization of zircaloy- 4 in $0.1 \mathrm{M}$ sulphamic acid

\begin{tabular}{lccc}
\hline Electrolyte & Formation Rate, V.s $^{-1}$ & $\mathrm{H}, \%$ & $\mathrm{~F}_{\mathrm{D}}$ in, $\mathrm{MV} . \mathrm{cm}^{-1}$ \\
\hline 0.1 M SA & 2.15 & 80.23 & 6 \\
0.1 M SA + 25 \% EG & 2.24 & 80.3 & 5.82 \\
0.1 M SA + 50 \% EG & 2.36 & 80.5 & 5.64 \\
0.1 M SA + 75 \% EG & 2.39 & 80.25 & 5.5 \\
\hline
\end{tabular}

$S A=$ Sulphamic acid, $E G=$ ethylene glycol, $\eta=$ current efficiency; $F_{D}=$ differential field of formation 
Table 2. Variation of dielectric constant as a function of solution composition

\begin{tabular}{cc}
\hline EG, $\%$ & Dielectric constant \\
\hline 0 & 80 \\
25 & 72.8 \\
50 & 69.2 \\
75 & 57.8 \\
\hline \multicolumn{2}{c}{ EG= ethylene glycol }
\end{tabular}

A further increase in the percentage of ethylene glycol to $80 \%$ results in non-uniformity in the film formation, which may be attributed to the fact that the electrolyte becomes highly non-polar due to the addition of more ethylene glycol. At high ethylene glycol concentrations, the mobility of the oxide ions (Which form the oxide film) decreases. Hence, in $80 \%$ ethylene glycol solution, the kinetics are slow. Though the dielectric constant value of the $>80 \%$ ethylene glycol solution is less, there is still poor film formation. This can be explained on the basis of the highly non-polar nature of the medium, which plays a major role in the anodization process.

\section{Effect of added anion impurities}

Experiments were carried out in $0.1 \mathrm{M}$ sulphamic acid $+0.001 \mathrm{M} \mathrm{SO}_{4}{ }^{2-}, \mathrm{PO}_{4}{ }^{3-}, \mathrm{CO}_{3}{ }^{2-}$ anions to check the effect of the added anions. The addition of phosphates, sulphates and carbonates resulted in improvement of the kinetics. The presence of phosphate ions improved the kinetics of anodization to better extent. It is observed that the oxide film formed on the alloy consisting of two discrete layers in $0.1 \mathrm{M}$ sulphamic acid appear as a single layer in presence of added anion impurity. The results are given in Table 3 and the plots are shown in Figures $3 \& 4$.

Table 3. Effect of added anion impurities

\begin{tabular}{lccc}
\hline Electrolyte & Formation rate,,.$^{-1}$ & $\eta, \%$ & $\mathrm{~F}_{\mathrm{D}}$ in, $\mathrm{MV} . \mathrm{cm}^{-1}$ \\
\hline $0.1 \mathrm{M} \mathrm{SA}$ & 2.15 & 80.23 & 6.00 \\
$0.1 \mathrm{M} \mathrm{SA}+0.001 \mathrm{M} \mathrm{CO}_{3}{ }^{2-}$ & 2.49 & 89.70 & 6.20 \\
$0.1 \mathrm{M} \mathrm{SA}+0.001 \mathrm{M} \mathrm{SO}_{4}{ }^{2-}$ & 2.52 & 98.72 & 5.70 \\
$0.1 \mathrm{M} \mathrm{SA}+0.001 \mathrm{M} \mathrm{PO}_{4}{ }^{3-}$ & 2.60 & 97.50 & 5.96 \\
\hline
\end{tabular}

$S A=$ Sulphamic acid $; \eta=$ current efficiency; $F_{D}=$ differential field of formation.

The increase in the kinetics when $0.001 \mathrm{M}^{\circ} \mathrm{PO}_{4}{ }^{3-}$ is added to $0.1 \mathrm{M}$ sulphamic acid can be explained by the firm incorporation of phosphate ions into the layers of the oxide films ${ }^{12}$. Added anions get incorporated between the ionic vacancy sites of the metal oxide films and reduce the height of the energy barrier of the movement of ions from one ionic site to another, thereby increasing the current. This incorporation increases the current efficiency with much ionic current getting utilized for film formation, which implies better kinetic results.

On the other hand, by the addition of $\mathrm{CO}_{3}{ }^{2-}$ and $\mathrm{SO}_{4}{ }^{2-}$, there is a little improvement in the kinetics, which implies that certain anions have favorable high influence on the kinetics of oxide film formation. Such ions that are capable of interstitial occupation in the film lattice increase the kinetics. 


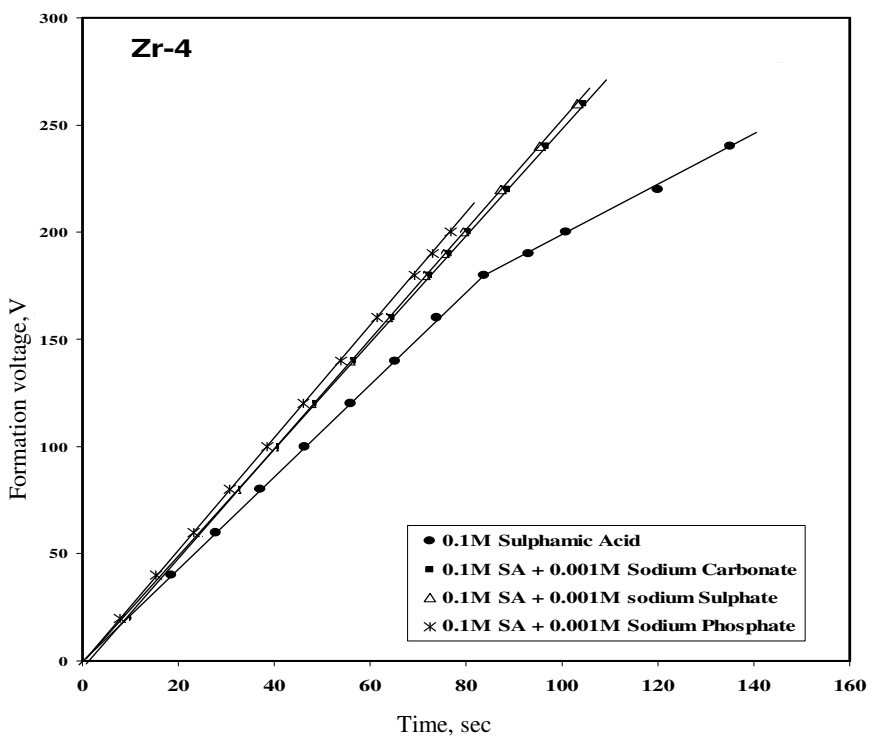

Figure 3. Plot of formation voltage as a function of time in anion impurity solution

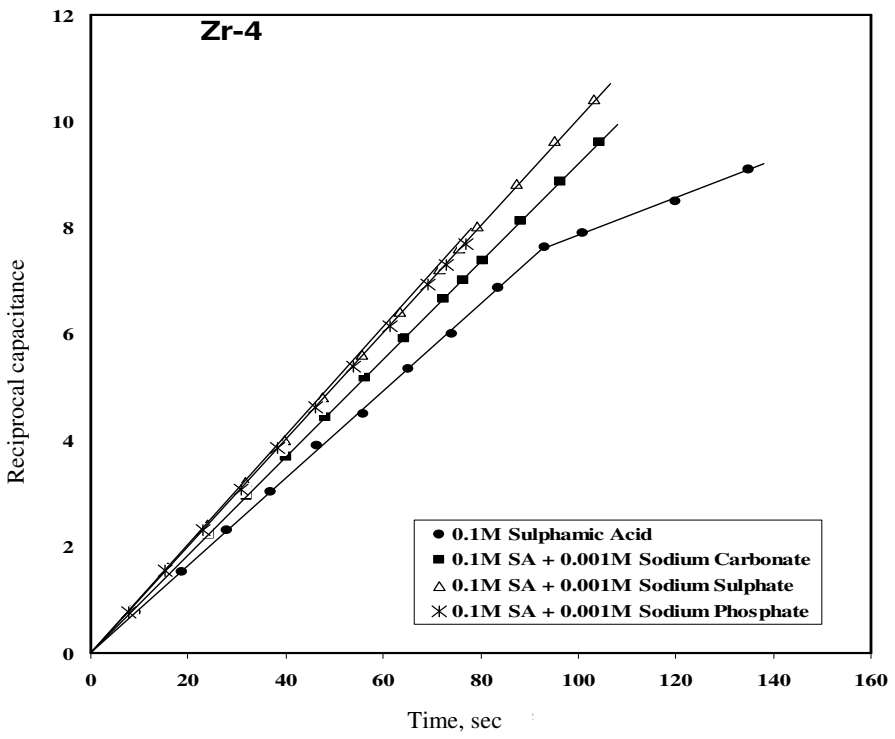

Figure 4. Plot of reciprocal capacitance as a function of time in anion impurity solution

\section{Conclusion}

For the anodization of zircaloy-4, the kinetics (formation rate, current efficiency) are better in $0.1 \mathrm{M}$ sulphamic acid with aquo-glycolic media and added anion impurities. Use of aquoglycolic media with up to $75 \%$ ethylene glycol showed improvement in the kinetics. Addition of phosphate anions in $0.001 \mathrm{M}$ concentration showed improvement in the kinetics. For the anodization of zircaloy-4 it can be concluded that the oxide film formed on the alloy consists of two discrete layers in $0.1 \mathrm{M}$ sulphamic acid and they are made to a single layer in presence of ethylene glycol and added anion impurity. 


\section{Acknowledgment}

The authors wish to thank Prof. Zubaida Azeem, Principal and Prof. Rafiq Mohiuddin, Head, Department of Chemistry, Osmania University College for Women for their keen interest and encouragement. The authors are also grateful to the NFC, Hyderabad for generous help in providing zircaloy-4. One of the authors (VDS) expresses his gratitude to Dr.B. Rajender, Principal, BJR Govt. Degree College, Nampally, Hyderabad for his encouragement.

\section{References}

1. Williams C D, Atomic Energy of Canada Limited Report, AECL 1969, 3456.

2. Williams C D, Reactor Technol., 1970, 13, 147.

3. Shobha Rani P, Anjaneyulu Ch and Sastry K.S, J Electrochem Soc India, 2000, 49(3), 129.

4. Lavanya A and Anjaneyulu Ch, Bull Electrochem., 2004, 20(4), 153.

5. Lavanya A, Raghunath G and Anjaneyulu Ch, Bull Electrochem., 2004, 20(7), 305.

6. Ashok Kumar V, Raghunath G and Anjaneyulu Ch, Bull Electrochem., 2005, 21(1), 17.

7. Lavanya and Anjaneyulu Ch, Bull Electrochem., 2002, 18, 317.

8. Arifuku F, Iwakura C, Yoneyama H and Tamura H, Denki Kagaski, 1978, 46, 19.

9. Randal J J, Electrochim Acta, 1975, 20, 663.

10. Lavanya A, Sarna C S N and Anjaneyulu Ch, J Electrochem Soc India, 2001, 50, 164.

11. Lavanya A, Raghunath Reddy G and Anjaneyulu Ch, Bull Electrochem., 2004, 20(7), 305-308.

12. Ashok Kumar V, Raghunath Reddy G and Anjaneyulu Ch, Bull Electrochem., 2005, 21, 17-21. 


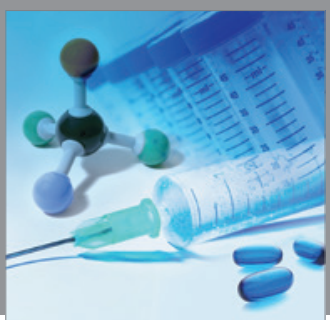

International Journal of

Medicinal Chemistry

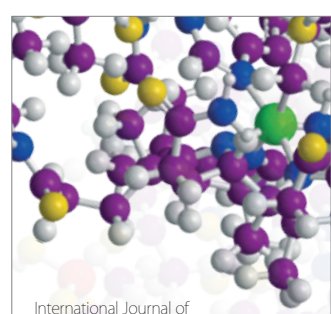

Carbohydrate Chemistry

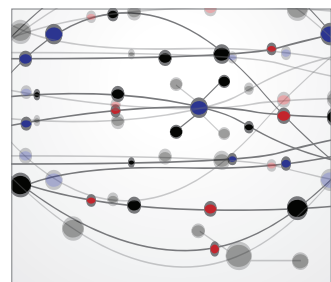

The Scientific World Journal
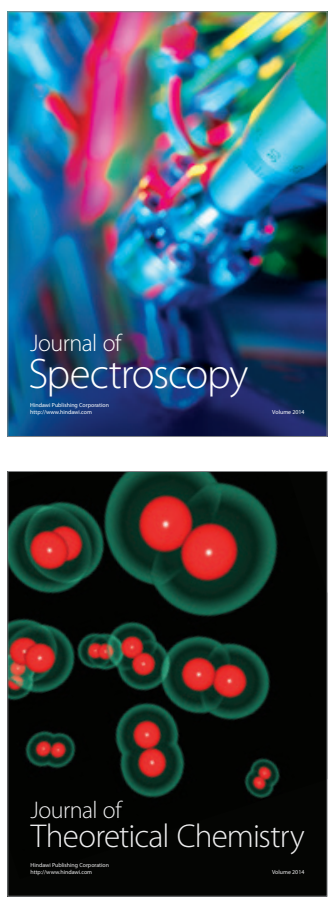
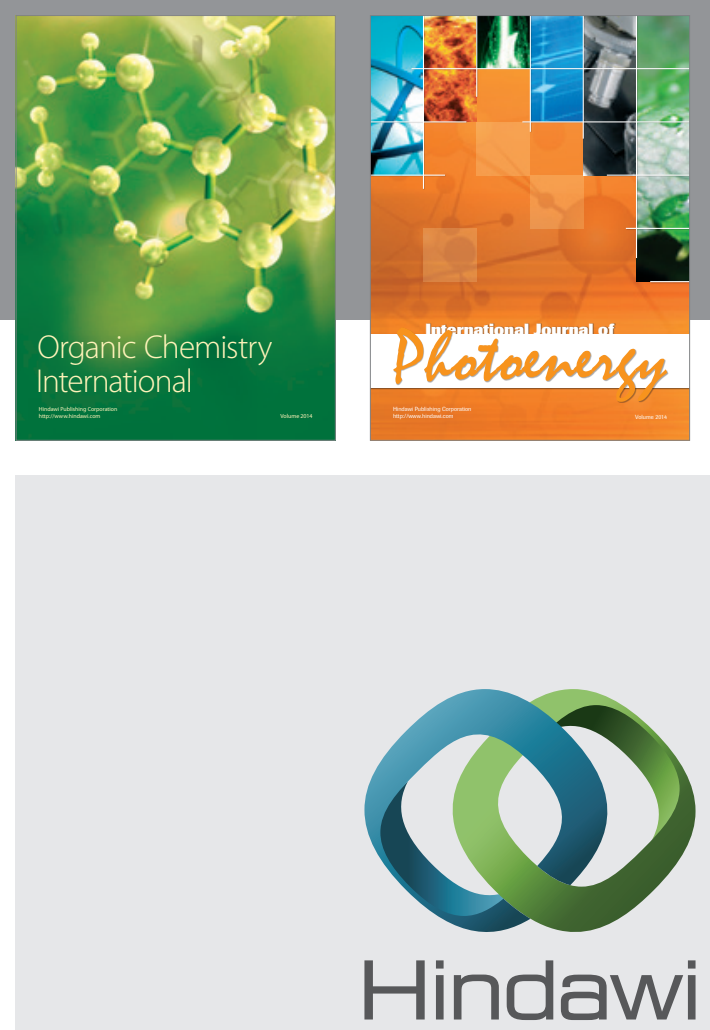

Submit your manuscripts at

http://www.hindawi.com
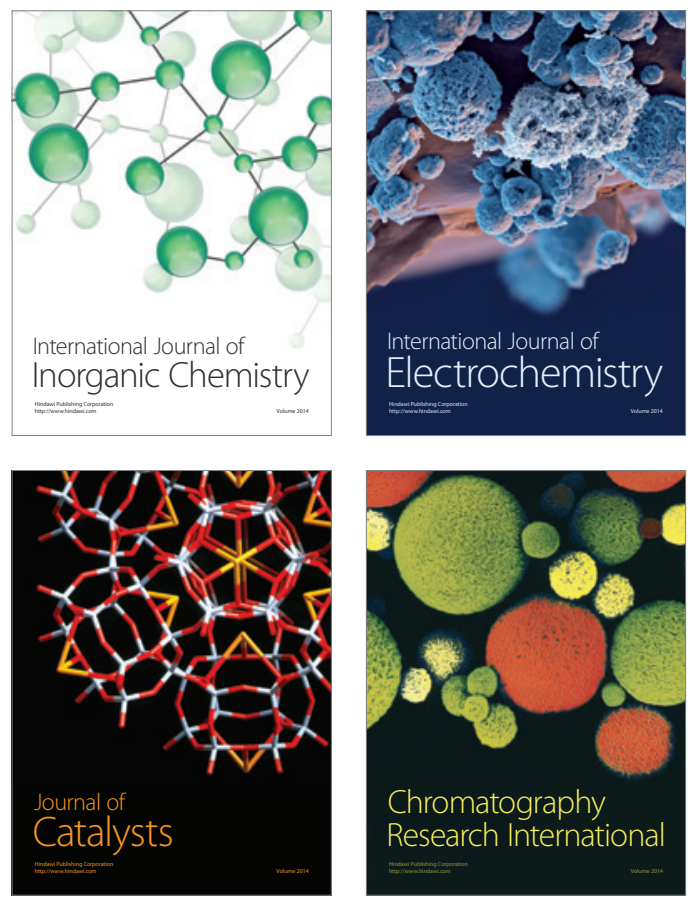
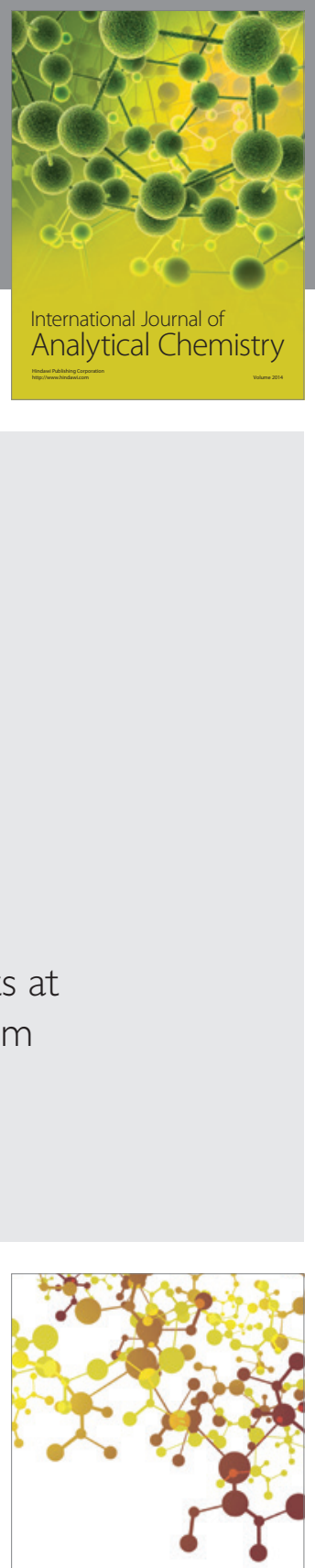

Journal of

Applied Chemistry
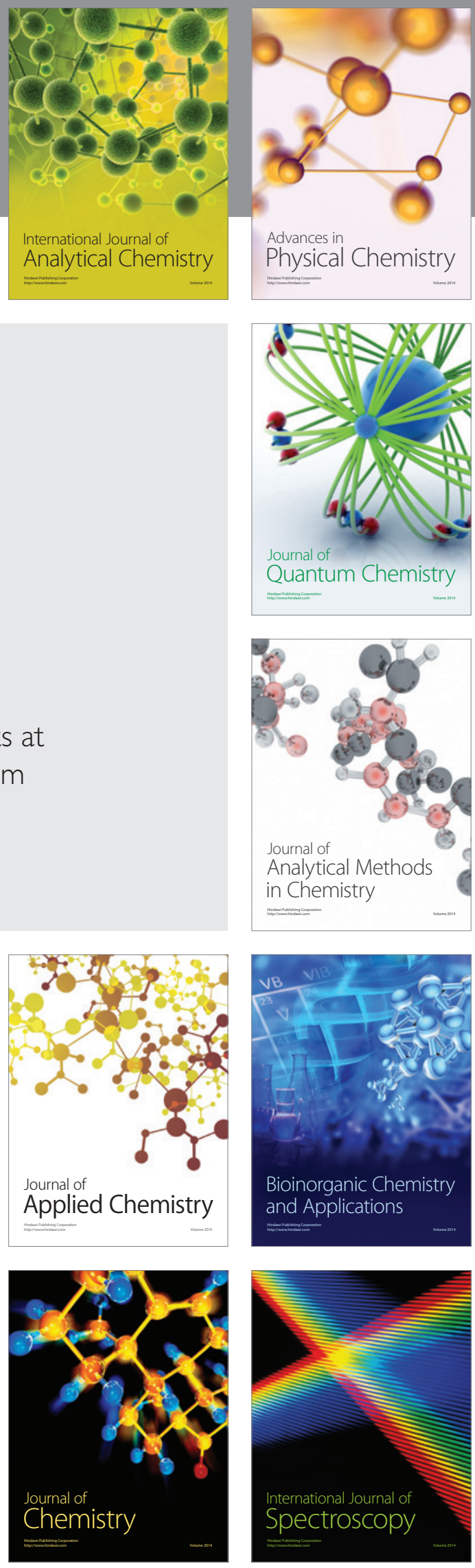